\title{
Outcome of complex non-unions of femoral fractures managed with llizarov method of distraction osteogenesis
}

\author{
Karim Bakhsh', Faridullah Khan Zimri², Atiq-Ur-Rehman ${ }^{3}$, \\ Eid Mohammad ${ }^{4}$, Muhammad Saaiq ${ }^{5}$
}

\begin{abstract}
ABSTARCT
Objective: To evaluate the management outcome of complex non-union of femoral fractures with llizarov method in terms of bone union, functional results and any complications.

Methods: This case series study was carried out at the Departments of Orthopedic Surgery, National Institute of Rehabilitation Medicine (NIRM), Islamabad and Civil hospital, Quetta over a period of three and half years, January 1, 2015 to June 30,2018.

Results: There were 50 patients in the study. There were $48(96 \%)$ males and $2(4 \%)$ females. The ages ranged between 17-54 years with a mean of 33.58 \pm 8.9 years. As per ASAMI criteria, the bone results were excellent in $17(34 \%)$, good in $30(60 \%)$, fair in $1(2 \%)$ and poor in $2(4 \%)$ patients. The functional results were excellent in $15(30 \%)$, good in $24(48 \%)$, fair in $8(16 \%)$ and poor in $3(6 \%)$. The bone union rate was $98 \%$ whereas infection eradication rate was $94 \%$. The most frequent complications were pin tract infection affecting $80 \%$ patients, knee stiffness $60 \%$ patients and K-wires loosening $20 \%$ patients.

Conclusion: The llizarov method provides an effective solution to address the complex non-union of femur fractures. It helps to ensure fracture healing, eradicates infection and provides good functional outcome. The attended complications are mild to moderate and manageable with conservative means.
\end{abstract}

KEYWORDS: Ilizarov technique, Ilizarov method, Non unions of femur fractures.

How to cite this:

doi: https://doi.org/10.12669/pjms.35.4.244

Bakhsh K, Zimri FK, Atiq-Ur-Rehman, Mohammad E, Saaiq M. Outcome of complex non-unions of femoral fractures managed with Ilizarov method of distraction osteogenesis. Pak J Med Sci. 2019;35(4):1055-1059.

doi: https://doi.org/10.12669/pjms.35.4.244

This is an Open Access article distributed under the terms of the Creative Commons Attribution License (http://creativecommons.org/licenses/by/3.0), which permits unrestricted use, distribution, and reproduction in any medium, provided the original work is properly cited.

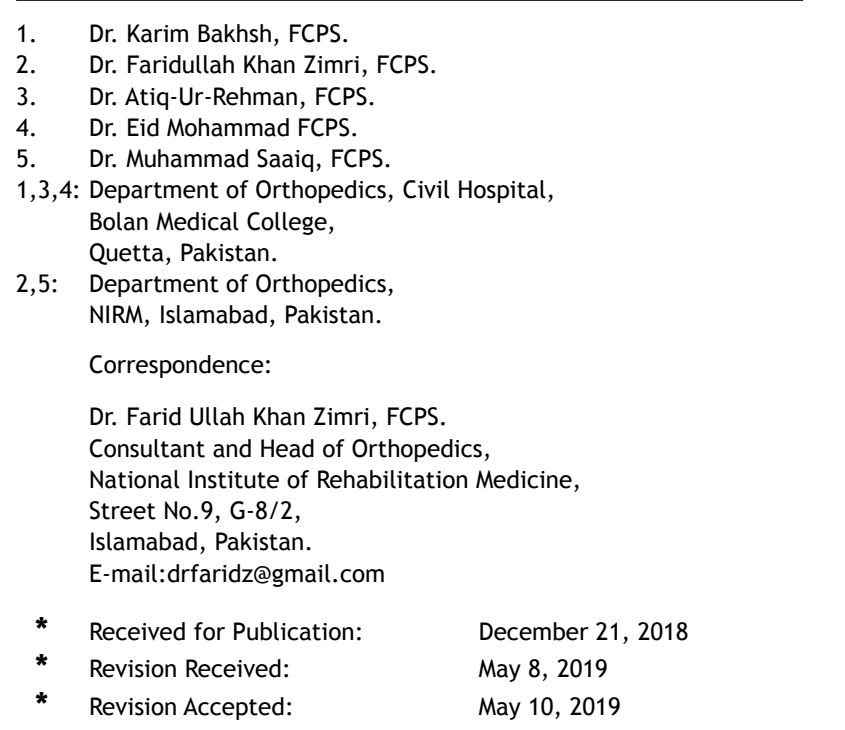

\section{INTRODUCTION}

Lower limb trauma and the associated skeletal injuries constitute a major workload of the orthopedic surgeons in developing countries like Pakistan. Fracture non-union of femur is one formidable issue which may be encountered in a significant proportion of these patients. Fractures may fail to progress to biological union owing to a host of underlying reasons. For instance, severe injuries with reduced arterial flow, associated soft tissue trauma, failure to ensure appropriate initial fracture management and osteomylitis. This may be further complicated by additional factors like bone loss, deformity and leg length discrepancy..$^{1-3}$

We employed Ilizarov method for managing non union of femur. This method is based on the fundamental principle of 'tension stress'. It involves 
gradual traction or stress on living tissues that induces regeneration and active growth of body tissues such as the bone, muscle, associated skin and soft tissues. The Ilizarov method has emerged as the gold standard for addressing the non union as well as associated bone loss, deformity and leg length discrepancy. ${ }^{3,4}$

The present study was carried out to document the management outcome of complex non unions of femur fractures managed with Ilizarov method in terms of bone results, functional outcome and any complications encountered during the course of treatment.

\section{METHODS}

We undertook this prospective descriptive case series study at the Departments of Orthopedic Surgery, National Institute of Rehabilitation Medicine (NIRM), Islamabad and Civil hospital, Bolan Medical College, Quetta over a period of three and half years from Jan 01, 2015 to June 30, 2018. The study protocol was approved by the ethics committees of the hospitals. Written informed consent was taken from the patients.

The study included all patients whose complex non unions of femoral fractures were managed with Ilizarov method. We defined complex non unions of femoral fractures as an established non-union (of at least 6-months duration) with one or more of the following criteria: a) infection at the site of nonunion, b) a bone defect $>4 \mathrm{~cm}$ and c) a previous failed attempt to achieve union with at least one supplementary intervention such as bone grafting or exchange nailing. Our exclusion criteria included patients not willing for prolonged treatment with Ilizarov method.

All the patients werehospitalized for management. They were initially evaluated with history, physical examination and baseline investigations. Standard $\mathrm{X}$-rays and tissue for culture sensitivity of the affected bone were performed. Standard wound care and antibiotic therapy was instituted according to the culture sensitivity reports of the wound specimens.

The surgeries were performed under spinal or general anesthesia. The patient was positioned supine on a radiolucent traction operating table. The ilizarov external fixator was applied in a standard fashion. We employed hybrid technique using combination of half pins and K-wires.

A bifocal compression distraction technique (compression of the non-union with distraction at the corticotomy) was employed in non-unions where the bone gap was $>2 \mathrm{~cm}$. Monofocal treatment (simple stabilization of the non-union and compression) was used in cases of non-unions where the gap was $>1-2 \mathrm{~cm}$. A standard bifocal circular frame for complex non-union of the femur consisted of 3-4 rings and proximal semicircular ring and arches. One week latency period was allowed before starting distraction. The rate of distraction was $1 \mathrm{~mm}$ per day, performed as $0.25 \mathrm{~mm}$ every six hourly. The ilizarov frame was retained until final union was achieved. Our definition of union was the presence of bridging trabeculae on 3 cortices, absence of pain on dynamization and the absence of movement at the union site when screened using fluoroscopy.

The Association for the Study and Application of Methods of Ilizarov (ASAMI) criteria was used to evaluate the bone results and functional results in our study. Various other outcome measurements included bone results, functional results and any complications encountered through the course of treatment.

We employed SPSS version 17 (SPSS Inc, Chicago, Illinois, USA) to carry out statistical analysis. Various descriptive statistics were used to calculate the outcome measures of interest.

\section{RESULTS}

There were 50 patients in the study, 48(96\%) males and 2(4\%) females, ages ranged between 1754 years with a mean of $33.58 \pm 8.9$ years. The various bacteria cultured from the infected bones included Staphylococcus aureus 40(80\%), Pseudomonas aeruginosa 17(34\%), Methicillin Resistant Staphylococcus aureus 8(16\%), Escherichia coli $8(16 \%)$ and various other bacterial species $7(14 \%)$. Twenty one wound cultures were positive for polymicrobials.

The time interval between the primary injury the definitive management with the Ilizarov method ranged from 10 to 20 months with a mean of 11 months. It was 9 months among 7(14\%) patients, 10 months among $15(30 \%)$ patients, 11 months among 19(38\%) patients, 12 months among 7(14\%) patients and 20 months among $2(4 \%)$ patients. At the time of presentation for definitive management with Ilizarov method, the surgical interventions already undergone by the patients included three surgeries among 27 (54\%)patients, two surgeries among $11(22 \%)$ patients, four surgeries among $9(18 \%)$ patients and one surgery among three $(6 \%)$ patients. The average number of surgeries were 
Table-I: Bone evaluation results among the patients. $(n=50)$.

\begin{tabular}{|c|c|c|c|}
\hline & $\begin{array}{l}\text { Bone } \\
\text { evaluation }\end{array}$ & Guidelines parameters & $\begin{array}{l}\text { Number/ } \\
\text { Percentage }\end{array}$ \\
\hline 1 & Excellent & Eradication of the infection, bone union, deformities $<7^{\circ}$ and LLD $<2.5 \mathrm{~cm}$ & $17(34 \%)$ \\
\hline 2 & Good & $\begin{array}{l}\text { Bone union }+ \text { any two of the following items eradication of infection, } \\
<7^{\circ} \text { deformity LLD }<2.5 \mathrm{~cm}\end{array}$ & $30(60 \%)$ \\
\hline 3 & Fair & $\begin{array}{l}\text { Bone union, persistence of infection, less than } 7^{\circ} \text { deformities and } \\
\text { more than } 2.5 \mathrm{~cm} \text { limb length discrepancies }\end{array}$ & $1(2 \%)$ \\
\hline 4 & Poor & Bone nonunion + infection + deformity $>7^{\circ}+$ LLD $>2.5 \mathrm{~cm}$ & $2(4 \%)$ \\
\hline
\end{tabular}

LLD: Limb length discrepancy.

2.84 per patient. Areas of the femur involved in the gap non union included distal femur $(n=31)$, mid femur $(\mathrm{n}=17)$ and proximal femur $(\mathrm{n}=2)$.

At the time of application of Ilizarov fixator, all patients had severe limitation of range of motion of the knee, with a mean $\mathrm{ROM}$ of $50^{\circ}$ (range, $0^{\circ}-70^{\circ}$ ). The mean ROM at the time of frame removal was $40^{\circ}$ (range, $20^{\circ}-90^{\circ}$ ). The mean ROM at 6 months following aggressive physiotherapy was $90^{\circ}$ (range, $\left.80^{\circ}-110^{\circ}\right)$.

Preoperatively the skeletal defects or bone gaps ranged from $1-7 \mathrm{~cm}$ with a mean of $3.62 \mathrm{~cm}$. It was $1 \mathrm{~cm}$ in $5(10 \%)$ patients, $2 \mathrm{~cm}$ in $7(14 \%)$ patients, $3 \mathrm{~cm}$ in $9(18 \%)$ patients, $4 \mathrm{~cm}$ in $16(32 \%)$ patients, $5 \mathrm{~cm}$ in $9(18 \%)$ patients, $6 \mathrm{~cm}$ in $2(4 \%)$ patients and 7 $\mathrm{cm}$ in $2(4 \%)$ patients. Postoperatively at the time of removal of frame, only $9(18 \%)$ patients had limb shortening of over $2.5 \mathrm{~cm}$. The mean shortening among them was $3.1 \mathrm{~cm}$ (range $3-4 \mathrm{~cm}$ ). Among all these cases further treatment was deliberately curtailed in order to reduce the duration of this complex treatment.

According to ASAMI criteria, bone results are displayed in Table-I whereas the functional results are shown in Table-II. Bone union was achieved among all except two patients. (98\% bone union rate). Infected was eradicated among $47(94 \%)$ patients. The bone transport time ranged from 29135 days with a mean of 65 days.

The complications encountered included pin tract infection among $40(80 \%)$ patients, knee stiffness among $30(60 \%)$ patients, K-wires loosening in
$10(20 \%)$ patients, delayed consolidation in $5(10 \%)$ patients, axial deviation during bone transport in $3(6 \%)$ patients, skin invagination requiring plastic surgical correction in $4(8 \%)$ patients, persistent non union in $2(4 \%)$ patients and femoral re fracture in $1(2 \%)$ patient. There was no mortality.

\section{DISCUSSION}

The Ilizarov method has stood the test of time for several decades in addressing a host of skeletal injuries and deformities. The technique was pioneered by the Russian inventor Ilizarov in 1951. The technique involves passing percutaneous wires, tensioning them adequately and attaching them to the rings of the circular metal frame. With this rigid arrangement, it is possible to simultaneously obtain fracture compression, bone distraction histiogenesis, bone distraction, bone lengthening and deformity correction. The fixator is strong enough to allow ambulation and immediate weight bearing. ${ }^{1-4}$

Prior to the development of the Ilizarov method, the treatment of bone loss and non-unions caused by traumatic injuries or infections involved the use of a variety of other surgical techniques such as the use of cancellous autogenous bone grafts, vascularized bone grafts, structural allografts and artificial bone substitutes, employment of internal fixation devices. The treatment of nonunions using the Ilizarov method depends on the type of nonunion, namely hypertrophic and

Table-II: Functional results among the patients. $(n=50)$.

\begin{tabular}{|c|c|c|c|}
\hline & $\begin{array}{l}\text { Functional } \\
\text { evaluation }\end{array}$ & Guidelines parameters & $\begin{array}{l}\text { Number/ } \\
\text { Percentage }\end{array}$ \\
\hline 1 & Excellent & $\begin{array}{l}\text { Active, no limp, minimal knee stiffness (loss of }<15^{\circ} \text { knee extension), no RDS, } \\
\text { and significant pain }\end{array}$ & $15(30 \%)$ \\
\hline 2 & Good & Active, no limp, pain significant, no RDS and $20^{\circ}$ loss of knee extension & $24(48 \%)$ \\
\hline 3 & Fair & Active, limping, knee stiffness, RSD and significant pain & $8(16 \%)$ \\
\hline 4 & Poor & Inactive and unable to return to daily activities & $3(6 \%)$ \\
\hline
\end{tabular}

RSD: Reflex sympathetic dystrophy. 
atrophic. Since the hypertrophic non-unions have sufficient vascularity to promote bone healing but lack the necessary structural stability, Ilizarov recommended gradual compression of the two surfaces of the nonunion to promote consolidation, followed by gradual distraction to compensate for the associated bone loss. In case of atrophic non-unions, there is significantly reduced bone healing potential, compression and distraction are carried out simultaneously at different locations in the affected bone. Generally speaking, the bone is compressed at the site of the nonunion and a separate osteotomy is performed at another part of bone fragment, and distraction is performed there. The two goals of treatment are achievement of fracture union and lengthening. These two can be split into two interventions sequentially. First surgery may be aimed to achieve union. The second intervention may be later instituted to correct the leg length discrepancy and any associated deformities.-8

In the majority of our patients the non union was associated with infection. We undertook an initial thorough debridement of the devitalized bone before instituting the distraction osteogenesis through Ilizarov method. Following the bone debridement a bone gap is created. The combination of the bone gap and associated infection precludes the potential use of an internal fixator. Hence we need an external fixation device. The Ilizarov method offers the most prudent option to address these issues as it on one hand provides stable fixation which is mechanically strong enough to allow mobilization and on other hand ensures new bone formation, lengthening and deformity correction. The technique helps to eliminate infection by increasing the local blood supply and vascularity of the osteomyelitic focus. Hence the famous notion "osteomyelitis burns in the fire of regeneration". Our favorable results with Ilizarov method for infected nonunion of the femur conform to several published studies..$^{9-12}$

In this study, we used the ASAMI criteria for bone evaluation and measuring the functional outcome. This system incorporates four main criteria for evaluating the bone results. These include bone/ fracture union, infection eradication, deformity correction and limb length discrepancy (LLD). The functional evaluation is based on five criteria. These include limp, joint stiffness, reflex sympathetic dystrophy, pain and inactivity. ${ }^{13}$

In our study the bone results were excellent in $34 \%$ and good in $60 \%$ patients. Our outcome results conform favorably to most of the published literature..$^{8,14-18}$ We had infection eradication among 94\% patients. Krishnan A et al. ${ }^{12}$ reported elimination of infection among nineteen of their twenty patients with infected non union of femur who were managed with the Ilizarov method.

In this study the functional results were excellent in $30 \%$ whereas good in $48 \%$ patients. Our outcome results conform favorably to the published studies. ${ }^{8,14-17}$ Krishnan A et al. ${ }^{12} 85 \%$ and $60 \%$ of patients had good-to-excellent bone and functional results, respectively..$^{5-8}$

In this study, the major share of complications was constituted by pin tract infection, knee stiffness and K-wires loosening. Krishnan $\mathrm{A}$ et al. ${ }^{12}$ reported re-fracture, femoral bowing of $20^{\circ}$ and shortening of $2.5 \mathrm{~cm}$, one each in their series. Palatnik $\mathrm{Y}^{4}$ reported. pain during the distraction phase, pintract infections and decreased range knee motion as their most common complications. Our findings conform to most of the published literature., ${ }^{4,8,19}$ Complications are intrinsic to the Ilizarov method however their frequency and severity decrease with increasing experience of the surgical team as well as improvement in the physiotherapy back up facility.

The Ilizarov method of treatment is attended by a host of challenges through the course of treatment. Firstly, it a relatively prolonged treatment and we initially counsel the patients specifically regarding the lengthy period of the fixator application. Also several complications are subsequently encountered through the course of treatment. For instance, pin tract infections, loosening of $\mathrm{K}$-wires, and knee stiffness. These issues often lead to compliance issues on part of the patients. These factors at times lead to deliberate shortening of the treatment and discontinuation before achievement of the desired leg length. ${ }^{4-8,16-21}$

Physiotherapy is an important adjunct to any good orthopedic surgical facility. Elsewhere in the world, physiotherapy has become an integral part of orthopedic surgery units. Unfortunately the process of integration of physiotherapy with orthopedic surgery is still in infancy in our country. Additionally compliance with home physiotherapy regimens is also very poor amongst our patients. Owing to these factors, we have higher rate of initial knee stiffness among our patients.

Most of the previously published local studies and literature on Ilizarov method have focused on the management of tibial fractures and correction of congenital deformities. ${ }^{18,20-22}$ Hence there is relative scarcity of similar local studies pertaining to the management of complex femoral non unions. Given the context of femoral fractures, the application 
of Ilizarov method is more demanding for the patient as well as the surgeon. The bulky muscular surroundings of the femur renders it a difficult bone to treat with this method. Transfixation of the local tissues is more frequently associated with pain and discomfort through the course of treatment. Additionally there is more frequent problem of knee stiffness and higher frequency of pin track problems. Our study should prompt future local multicenter studies to confirm and improve upon our study. This will help to generate local evidence base for better management of patients with complex non unions of femoral fractures.

\section{CONCLUSION}

The Ilizarov method provides an effective solution to address the complex non-union of femur fractures. It helps to ensure fracture healing, eradicates infection and provides good functional outcome. The attended complications are mild to moderate and manageable with conservative means.

Conflicts of Interest: None declared.

Financial Disclosure: None declared.

\section{REFERENCES}

1. Yin $\mathrm{P}, \mathrm{Ji} \mathrm{Q}, \mathrm{Li} \mathrm{T}, \mathrm{Li} \mathrm{J}, \mathrm{Li} \mathrm{Z}$, Liu J, et al. A systematic review and meta-analysis of Ilizarov methods in the treatment of infected nonunion of tibia and femur. PLoS ONE. 2015;10(11):e0141973. doi: 10.1371/journal.pone.0141973.

2. DharSA, Mir MR, Ahmed MS, AfzalS, Butt MF, Badoo AR, et al. Acute peg in hole docking in the management of infected non-union of long bones. Int Orthopaed. 2008;32(4):559-566. doi: 10.1007/s00264-007-0353-6.

3. Pande H, Singh CM, Prabhakara A, Philip VM, Iqbal MS, Sharma Y. Management of infected non-union of lower limb long bone fractures using ilizarov technique: a study of outcomes. Int J Res Orthop. 2017;3(3):557-564. doi: 10.18203/ issn.2455-4510.IntJResOrthop20171901.

4. Palatnik Y, Rozbruch SR. Femoral reconstruction using external fixation. Adv Orthoped. 2011. doi: 10.4061/2011/967186.

5. Lai D, Chen CM, Chiu FY, Chang MC, Chen TH. Reconstruction of juxta-articular huge defects of distal femur with vascularized fibular bone graft and Ilizarov's distraction osteogenesis. J Trauma. 2007;62(1):166-173. doi: 10.1097/TA.0b013e31802dccdb.

6. Zhang X, Liu T, Li Z, Peng W. Reconstruction with callus distraction for nonunion with bone loss and leg shortening caused by suppurative osteomyelitis of the femur. J Bone Joint Surg Br. 2007;89(11):1509-1514. doi: 10.1302/0301-620X.89B11.19239.

7. Morasiewicz L, Orzechowski W, Kulej M, Stepniewski $M$. The results of treatment of bone defects and nonunion within the femoral shaft with shortening of femur using the Ilizarov method. Ortop Traumatol Rehabil. 2007;9(4):366-376.
8. Aronson J, Limb-lengthening, skeletal reconstruction, and bone transport with the Ilizarov method. J Bone Joint Surg Am. 1997;79(8):1243-1258.

9. Song HR, Kale A, Park HB, Koo KH, Chae DJ, Oh CW, et al. Comparison of internal bone transport and vascularized fibular grafting for femoral bone defects. J Orthop Trauma. 2003;17:203-211.

10. Barbarossa V, Matkovic BR, Vucic N, Bielen M, Gluhinic M. Treatment of osteomyelitis and infected non-union of the femur by a modified Ilizarov technique: follow-up study. Croat Med J. 2001:42;634-641.

11. Gualdrini G, Stagni C, Fravisini M, Giunti A. Infected nonunion of the femur. Chir Organi Mov. 2002;87:225-233.

12. Krishnan A, Pamecha C, Patwa JJ. Modified Ilizarov technique for infected nonunion of the femur: the principle of distraction-compression osteogenesis. J Orthop Surg. 2006;14(3):265-272. doi: 10.1177/230949900601400307.

13. Paley D, Catagni MA, Argnani F, Villa A, Benedetti GB, Cattaneo R. Ilizarov treatment of tibial non-unions with bone loss. Clin Orthop Relat Res. 1989;241:146-165.

14. Zaidi IH, Sahito B, Tariq SM, Kumar D, Arsalan, Ali M. Outcome of nonunion femur with Ilizarov technique. J Surg Pak. 2015;20(4):147-150.

15. Moustafa MS, Radwan MA. Functional and radiological evaluation of treatment of infected nonunited fracture shaft femur using Ilizarov external fixator. Egypt Orthop J. 2015;50:139-143. doi: 10.4103/1110-1148.177914.

16. Hakeem A, Azim Q, Ali A, Mufti AJ, Essa A, Ali I. Management of Infected nonunion of long bones, a case series. J Pak Orth Assoc. 1918;30(2):1-7.

17. Shamim A, Chaudhry FA, Mehmood A. Use of Ilizarov method in the treatment of non-unions, infected nonunions, infected non-unions with bone defects, malunions, infected malunions, infected/ open fractures of the femur. Annals King Edward Med Coll. 2002;8(1):22-24.

18. Bakhsh K, Rehman AU, Zimri FK, Mohammad E, Ahmed W, Saaiq M. Presentation and management outcome of tibial infected non-union with Ilizarov technique. Pak J Med Sci. 2019;35(1):136-140. doi: 10.12669/pjms.35.1.67

19. Inan M, Karaoglu Cilli FC, Turk CY, Harma A. Treatment of femoral nonunions by using cyclic compression and distraction. Clin Orthop Rel Res. 2005;436:222-228. doi: 10.1097/01.blo.0000159153.09508.97.

20. Iqbal $P$, Afaq $M$, Qayum H. Treatment of tibial non-union with the Ilizarov method. Proceedings Sheikh Zayed. Posdgrad Med Inst. 1999;13(1-2):71-75.

21. Khan MS, Raza W, Ullah H, Khan AS, Siraj, M, Askar Z. Outcome of Ilizarov fixator in complex non-union of long bones. J Pak Med Assoc. 2015;65(11 Suppl 3):S147-151.

22. Saaiq M, Ashraf B. From reconstructive triangle to reconstructive quadrangle: Time to include tissue distraction in the reconstructive algorithms. World J Plast Surg. 2016;5(2):185-186.

\section{Authors' Contributions:}

FUKZ, KB, AUR \& MS: designed the study and prepared the manuscript.

MS, EM \& AUR: Performed data collection and analyzed the results.

All authors approved the manuscript. 